\title{
A metallic magnetic calorimeter dedicated to the spectrometry of L X-rays emitted by actinides
}

\author{
Matias Rodrigues $^{\mathrm{a}}$, Riham Mariam, and Martin Loidl \\ CEA, LIST, Laboratoire National Henri Becquerel (LNE-LNHB), Bât. 602 PC111, CEA-Saclay, 91191 Gif-sur-Yvette Cedex, France
}

\begin{abstract}
Many actinides emit intense L X-rays consecutively to their decay. However the intensities of these $\mathrm{X}$-rays are not well known: they are generally calculated with relatively large uncertainties and do not always agree with existing measurements. The latter ones are obtained with semiconductor spectrometers, but due to their insufficient energy resolution, these detectors are not able to separate the many X-ray lines and to give detailed emission intensities. So new measurements of precise and detailed L X-ray emission intensities are required. These would be beneficial on the one hand for the knowledge of the decay schemes and on the other hand as reference data for end-users of X-ray spectrometry. Therefore a spectrometer with a high energy resolution has been developed based on the technology of metallic magnetic calorimeters. The L X-ray spectra from ${ }^{241} \mathrm{Am}$ and ${ }^{210} \mathrm{~Pb}$ decays show a FWHM energy resolution of $26 \mathrm{eV}$ associated with a constant detection efficiency between 5 and $26 \mathrm{keV}$. With such performance, about 30 relative L X-ray intensities can be determined for ${ }^{241} \mathrm{Am}$ and ${ }^{210} \mathrm{~Pb}$. The measured emission intensities of $\mathrm{L} \mathrm{X}$-ray groups are compared with those published as obtained with conventional techniques; the good agreement between the data validated our technique.
\end{abstract}

\section{X-ray emission intensities of actinides}

More accurate decay data of heavy elements and actinides are beneficial for fission reactors (safety, waste management...), for nuclear safeguards, for the environmental survey of specific actinides and their decay chains; improved decay data also extend the scientific knowledge for physics research and non-energy applications.

X-ray emission intensities are part of the decay data. Actinides are particularly relevant because most of them emit intense L X-rays, ranging from a few photons per 100 decays up to one hundred (Table 1 ). These actinides decay by alpha or beta minus emission and the daughter nuclei de-excite through many gamma transitions. Some intense transitions have low energies $(<100 \mathrm{keV})$ and are strongly converted, meaning that many atomic vacancies are created in the L shells or in the outer shells, followed by X-ray emission during the atomic rearrangement. Even though the L X-ray emission intensities are quite intense they are not experimentally precisely known.

Table 1 summarizes, for some actinides, the $\mathrm{X}$-ray emission intensities extracted from decay data evaluations [1,2]. The majority of them are calculated. The calculations have larger uncertainties than recent measurements, and they do not always agree. The calculations require many fundamental parameters (FPs) both nuclear (internal conversion coefficients (ICCs), transition probabilities) and atomic (fluorescence yield, Coster Kronig transition probabilities, emission rates...) and some of these have large uncertainties, in particular the atomic FPs. Also calculations are subject to important systematic errors because the calculated FPs are based on some assumptions (e.g., based on single vacancy configurations); or it is assumed that the gamma levels are balanced and consistent, and that the theoretical ICCs can be used, i.e., there are no anomalous $\gamma$-transitions.

There are only a small number of measurements published compared to the number of actinides and these measurements cover only the L X-ray groups, i.e., $\mathrm{L} \iota, \mathrm{L} \alpha, \mathrm{L} \eta, \mathrm{L} \beta$ and $\mathrm{L} \gamma$; they do not detail the $\mathrm{L}$ $\mathrm{X}$-ray emission intensities of individual lines. Precise and detailed measurements are difficult because:

- L X-ray spectra are complex, tens of X-ray transitions are concentrated in a narrow energy range of about $15 \mathrm{keV}$ and conventional detector technology, i.e., a semiconductor spectrometer, is not able to separate the many X-ray lines;

- In addition the detection efficiency of semiconductor detectors varies by more than $15 \%$ in the energy range of interest due to the presence of entrance windows, dead layers, and electrodes. An efficiency calibration is needed with standard sources, but there is a lack of such standard sources in this energy range;

- Spectral interferences in the L X-ray spectrum can occur due to the decays of isotopic impurities and/or the daughter.

A better and more detailed knowledge of L X-ray emission intensities would be beneficial:

- it would provide a better knowledge of the decay schemes of the actinides by verifying the consistency between the $\gamma$-transition probabilities, the $\gamma$-emission probabilities and the ICCs. In this

a e-mail: matias.rodrigues@cea.fr

(C) The Authors, published by EDP Sciences. This is an Open Access article distributed under the terms of the Creative Commons Attribution License 4.0 (http://creativecommons.org/licenses/by/4.0/). 
Table 1. List of some actinides with their evaluated L X-ray emission intensities and the method to determine them [1,2].

\begin{tabular}{lcclcc}
\hline Nuclide & Daughter & $\begin{array}{c}\text { L X-ray } \\
\text { intensity } \\
(\%)\end{array}$ & Method & $\begin{array}{c}\text { Number } \\
\text { of } \\
\text { measurements }\end{array}$ & $\begin{array}{c}\text { Agreement } \\
\text { Calculation/ } \\
\text { measurements }\end{array}$ \\
\hline Pa-231 & Ac-227 & $44.3(13)$ & calculated & 1 & agree \\
U-236 & Th-232 & $9.4(10)$ & calculated & - & - \\
U-237 & Np-237 & $59.0(21)$ & calculated & - & - \\
U-238 & Th-234 & $7.94(28)$ & calculated & - & - \\
$\mathrm{Pu}-238$ & $\mathrm{U}-234$ & $10.63(8)$ & Measured & 4 & agree \\
$\mathrm{Pu}-239$ & $\mathrm{U}-235$ & $4.66(5)$ & Measured & 4 & disagree \\
$\mathrm{Pu}-240$ & $\mathrm{U}-236$ & $10.34(15)$ & Measured & 6 & agree \\
$\mathrm{Pu}-242$ & $\mathrm{U}-238$ & $8.71(21)$ & calculated & - & - \\
$\mathrm{Am}-241$ & $\mathrm{~Np}-237$ & $37.66(17)$ & Measured & 9 & disagree \\
$\mathrm{Am}-242$ & $\mathrm{Pu}-242$ & $10.8(5)$ & calculated & - & - \\
$\mathrm{Am}-243$ & $\mathrm{~Np}-239$ & $18.9(7)$ & calculated & - & - \\
$\mathrm{Cm}-242$ & $\mathrm{Pu}-238$ & $9.92(23)$ & calculated & 2 & disagree \\
$\mathrm{Cm}-243$ & $\mathrm{Pu}-239$ & $52.1(16)$ & calculated & - & - \\
$\mathrm{Cm}-244$ & $\mathrm{Pu}-240$ & $8.92(23)$ & calculated & 1 & agree \\
\hline
\end{tabular}

case the radiative intensities of the subshells $\mathrm{L}_{1}$, $\mathrm{L}_{2}$ and $\mathrm{L}_{3}$ are needed, which in turn requires the detailed emission intensities since the $\mathrm{L} \beta$ and $\mathrm{L} \alpha$ groups are a mixture of $\mathrm{L}_{1}, \mathrm{~L}_{2}$ and $\mathrm{L}_{3} \mathrm{X}$-ray transitions;

- In case of anomalous $\gamma$ transitions the X-ray intensities are required to calculate the correct ICCs [3]; the subshells radiative intensities are equally needed.

- It will provide reliable data for the analysis of the $\mathrm{L}$ $\mathrm{X}$-ray spectra by end-users;

Spectrometers with improved energy resolution are indispensable to separate the many L X-ray lines and measure their emission intensities. Therefore a dedicated ultra-high energy resolution spectrometer, called SMX3, based on Metallic Magnetic Calorimeter (MMC) technology has been developed.

\section{Experimental set-up}

\subsection{The physical principle of MMCs}

MMCs are thermal cryogenic detectors, i.e., the energy $E$ deposited by the particle is measured as a temperature elevation given by $\Delta T=E / C$, with $C$ the detector heat capacity [4]. The detector is composed of an absorber, where the photon interacts, strongly thermally coupled with a thermal sensor measuring the temperature elevation. The MMC sensor is a paramagnetic material with a magnetization strongly dependent on the temperature. The sensor is placed on a superconducting flux transformer consisting of a meander shaped pick-up coil and a SQUID (Superconducting QUantum Interference Device) input coil (Fig. 1). A SQUID and its electronics precisely convert the flux change through the input coil into a voltage variation. After each particle interaction, the detector recovers its equilibrium temperature with a decay time constant $\tau=C / G$ imposed by a thermal link between the detector and the thermostatic bath. $G$ is the thermal conductance of the link. Toward very low temperature, the signal size is maximized because the detector heat capacity is minimized, and the main sources of noise decrease, so very high energy resolution can be obtained below $30 \mathrm{mK}$.

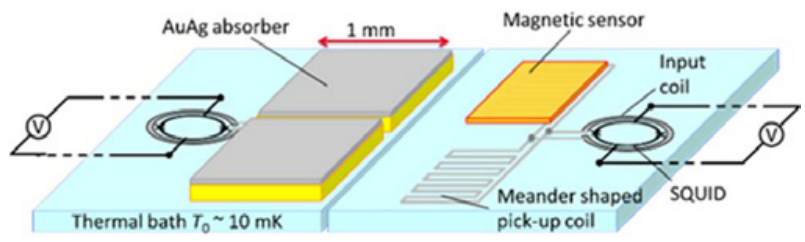

Figure 1. Scheme of the MMC using meander pick-up coils.

\subsection{The dedicated MMC}

The conception of a thermal cryogenic detector is a compromise between absorber volume (detection efficiency), decay time constant (count rate) and signal to noise ratio (energy resolution): large efficiency and high count rate are preferable for spectrometry applications, but large absorber volumes and fast decay time degrade the resolution [4].

\subsubsection{Detection efficiency and count rate}

Ideally the intrinsic detection efficiency is as constant as possible with the photon energy in order to achieve a precise and simple efficiency calibration, since it is one of the main contributions to the uncertainty of the emission intensities. For this purpose, a special bi-layer $\mathrm{Au}-\mathrm{Ag}$ absorber has been developed; all the photons below $25 \mathrm{keV}$ are absorbed in a $50 \mu \mathrm{m}$ thick Au layer; the role of the $\mathrm{Ag}$ layer of $17 \mu \mathrm{m}$ facing the source is to reabsorb the escape photons from the Au layer [5]. Such an absorber has an intrinsic efficiency varying less than $0.9 \%$ between 9 and $24 \mathrm{keV}$ (Fig. 2). In Fig. 2, the simulated intrinsic efficiency is compared with experimental data measured with ${ }^{241} \mathrm{Am}$ and ${ }^{210} \mathrm{~Pb}$ sources. A good agreement is observed and validates the simulated efficiency.

In comparison, semiconductor spectrometers, $\mathrm{Si}(\mathrm{Li})$ or HPGe, have an efficiency varying typically by $19 \%$ and $15 \%$ respectively, due to the escaping fluorescence photons, the dead layer, entrance windows, or the transmission through the absorber.

For $\alpha$-particle emitters, a Be window of $40 \mu \mathrm{m}$ thickness is placed between the MMC and the source to stop the $\alpha$-particles. Such a window affects the efficiency by only $0.5 \%$ between 9 and $24 \mathrm{keV}$. 


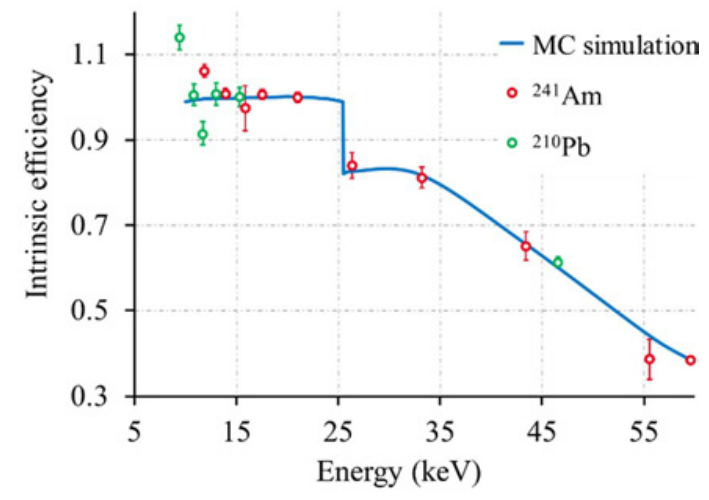

Figure 2. Intrinsic detection efficiency of the bi-layer absorbers of SMX3 obtained from Monte Carlo simulations (line). The circle points are experimental data derived from the tabulated emission intensities for ${ }^{241} \mathrm{Am}$ and from [8] for ${ }^{210} \mathrm{~Pb}$.

Due to the constraint on the absorber volume to achieve a high energy resolution, its area has been limited to $\approx 1 \mathrm{~mm}^{2}$. In order to increase the solid angle, an array of 4 absorbers has been built, the total absorber area is $\approx 4 \mathrm{~mm}^{2}$. Two sensors with their absorbers are read by two pick-up coils gradiometrically connected and read out by a single SQUID (Fig. 1). The gradiometric configuration cancels signal baseline fluctuations from bath temperature fluctuations and parasitic external magnetic fields. The two absorbers give opposite pulse polarities [6].

For quantitative and metrological measurements, L $\mathrm{X}$-ray spectra with statistics of a few million counts are required. The acquisition time is not a key constraint for the present application (except for radionuclides with short half-lives). If the spectrum can be acquired during a number of weeks, a total count rate $<10 \mathrm{~s}^{1}$ is enough, i.e., $2.5 \mathrm{~s}^{1}$ per absorber and a decay time constant of a few $\mathrm{ms}$ is sufficient. The thermal link has been designed for a $\tau_{d}=4 \mathrm{~ms}$.

\subsubsection{Figures of merit of the energy spectrum}

The relevant figures of merit in a complex energy spectrum as for L X-rays are high energy resolution, and a simple response function with a Gaussian shape at the full energy peak (FEP). These figures of merit were obtained from two spectra of ${ }^{241} \mathrm{Am}$ (Fig. 3) and ${ }^{210} \mathrm{~Pb}$ sources measured with SMX3.

For photons below $25 \mathrm{keV}$, the response function of SMX3 is nearly given by the FEPs because the intrinsic efficiency is close to unity; other spectral components, such as escape peaks and background, are negligible. For photons above $25 \mathrm{keV}$, the response function is more complex because there are the escape peaks of K X-rays from the Ag layer, which may interfere with the L X-ray FEPs. However, for most of actinides, $\gamma$-rays and $\mathrm{K} \mathrm{X}$-rays above $25 \mathrm{keV}$ have weak emission probabilities.

The spectrum from each absorber has a constant FWHM energy resolution below $26 \mathrm{keV}$, but are slightly different from one another (from 23.5 to $25.9 \mathrm{eV}$ ). The spectra have small and also slightly different nonlinearities (less than $0.13 \%$ between 6.4 and $59.5 \mathrm{keV}$ ). In order to obtain a Gaussian response for the FEPs in the summed spectrum, the energy resolutions have to be degraded to the worst one (i.e., $25.9 \mathrm{eV}$ ) and the

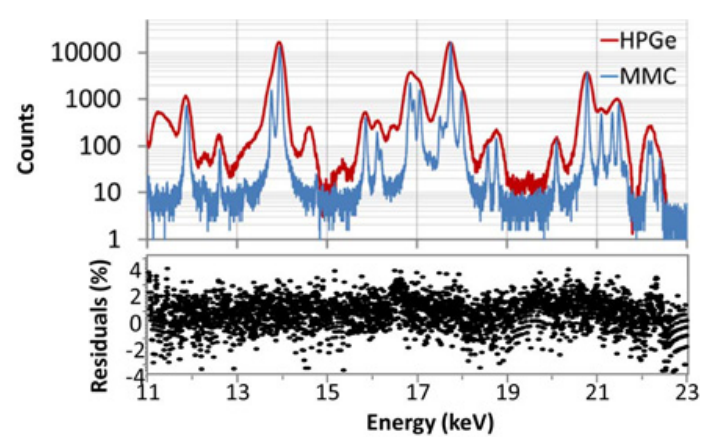

Figure 3. Upper part, comparison between the spectra of the L $\mathrm{X}$-rays from ${ }^{241} \mathrm{Am}$ obtained with a conventional planar HPGe detector (red) and with the MMC SMX3 (blue). Lower part, residuals between the spectrum obtained with SMX3 and its fit (not shown) with Voigt functions.

non-linearities have to be corrected for each absorber spectrum.

The L X-ray FEPs are fitted with Voigt functions, the Lorentzian widths are fixed parameters according to the level widths evaluated by Campbell and Papp [7], and the Gaussian width corresponds to the broadening of the detector response at the FEP (FWHM of $25.9 \mathrm{eV}$ ). In Fig. 3, looking at the residuals between the fit and the experimental spectrum, no structure appears, meaning that the MMC SMX3 has a Gaussian response at the FEPs.

\section{Results}

The L X-ray spectra of ${ }^{241} \mathrm{Am}$ and ${ }^{210} \mathrm{~Pb}$ were recorded for about 10 days, each at a temperature of about $10 \mathrm{mK}$ in a dilution refrigerator. They have counting statistics of $1.3 \times$ $10^{6}$ and $4.3 \times 10^{6}$ from which 32 and $28 \mathrm{~L} \mathrm{X}$-ray relative emission intensities have been measured respectively for ${ }^{241} \mathrm{Am}^{1}$ and ${ }^{210} \mathrm{~Pb}$ [8]. There are more than three orders of magnitude between the strongest and weakest lines (i.e., for ${ }^{241} \mathrm{Am} \mathrm{L}_{3}-\mathrm{M}_{5}$ and $\mathrm{L}_{1}-\mathrm{O}_{4,5}$ ).

The uncertainties on the measured emission intensities take into account the uncertainty for the efficiency correction (obtained by Monte Carlo simulation), the counting statistics and the fitting procedure. The most intense and well separated lines have low uncertainties dominated by the contribution of the efficiency correction, e.g., the $\mathrm{L}_{3}-\mathrm{M}_{5}$ intensity has a relative uncertainty of $0.26 \%$, while the weak lines, which merge and overlap with other lines, have larger uncertainties dominated by the contributions from the fitting procedure and the counting statistics; e.g., $\mathrm{L}_{1}-\mathrm{O}_{4,5}$ has a relative uncertainty of $22 \%$.

Absolute emission intensities have been derived by normalizing the relative L X-ray emission intensities with a reference line with a known absolute emission intensity. For ${ }^{241} \mathrm{Am}$ and ${ }^{210} \mathrm{~Pb}$, the $\gamma$-emission at $59.54 \mathrm{keV}$ and $46.54 \mathrm{keV}$, respectively, and some L X-ray groups are well-known and can be used as reference lines. Therefore the uncertainties of the absolute emission intensities have to take into account the uncertainties of the reference line emission intensity and the efficiency correction for the reference line energy. Such a normalization is not possible for actinides with unprecise emission intensities. Therefore

\footnotetext{
${ }^{1}$ The L X-ray emission intensities of ${ }^{241} \mathrm{Am}$ will be published in another journal.
} 
Table 2. Comparison between published L X-ray emission intensities and those measured with the MMC SMX3. The red values are intensities in disagreement and the blue ones are in agreement with our measurements.

\begin{tabular}{l|l|llllll}
\hline Radio-nuclide & Reference & $\mathbf{L} \iota$ & $\mathbf{L} \alpha$ & $\mathbf{L} \eta$ & $\mathbf{L} \beta$ & $\mathbf{L} \gamma$ & Total \\
\hline \multirow{2}{*}{${ }^{210} \mathrm{~Pb}$} & Schotzig [9] & $0.55(3)$ & $9.48(17)$ & $0.075(4)$ & $10.9(4)$ & $2.36(5)$ & $23.4(4)$ \\
& Rodrigues [8] & $0.518(10)$ & $9.49(19)$ & $0.087(2)$ & $10.52(21)$ & $2.320(35)^{*}$ & $23.01(44)$ \\
& Present work & $0.593(9)$ & $9.52(14)$ & $0.0825(12)$ & $10.58(16)$ & - & $23.11(40)$ \\
\hline \multirow{3}{*}{${ }^{241} \mathrm{Am}$} & Cohen [10] & $0.83(3)$ & $12.7(4)$ & $0.368(5)$ & $18.3(6)$ & $4.8(2)$ & - \\
& Bland [11] & $0.837(10)$ & $13.01(10)$ & $0.377(15)$ & $18.61(15)$ & $4.815(38)$ & $37.65(19)$ \\
& Lépy [12] & $0.864(10)$ & $13.03(13)$ & $0.369(12)$ & $18.39(19)$ & $4.74(8)$ & - \\
& Chechev [13] & $0.844(9)$ & $13.02(10)$ & $0.384(20)$ & $18.58(13)^{*}$ & $4.83(3)$ & $37.66(17)$ \\
& Present work & $0.893(11)$ & $13.08(14)$ & $0.373(5)$ & - & $4.79(6)$ & $37.73(28)$ \\
\hline
\end{tabular}

the absolute efficiency of the MMC SMX3 will soon be calibrated for future measurements.

Semiconductor spectrometers are not able to measure many L X-ray emission intensities - only emission intensities of L X-ray groups are determined. Table 2 shows the intensities of the L X-ray groups from the most recent measurements and from evaluated decay data, compared with our results. Good agreement is observed for the most intense groups, validating our measurement technique, as well as the efficiency correction and the spectrum analysis.

\section{Conclusions}

An MMC, SMX3, with ultra-high energy resolution (FWHM of $26 \mathrm{eV}$ ) has been developed and optimized for measuring the L X-ray emission intensities of actinides. It has a constant efficiency between 5 and $25 \mathrm{keV}$ that minimizes the efficiency uncertainty. For the lines with the highest intensities (i.e., $L \alpha_{1}$ ) a relative uncertainty of few $\%_{0}$ is achievable on the relative intensities. The comparison with the published emission intensities of the L X-ray groups shows a good agreement and validates the entire experimental procedure. The determination of absolute emission intensities is possible for actinides having at least one well known emission intensity which can be used as a reference line. It is planned to calibrate the absolute efficiency in order to be able to measure absolute emission intensities for other actinides. In addition, the detector SMX3 will continue to measure L X-ray spectra of actinides such as $\mathrm{Pu}$ isotopes or ${ }^{233} \mathrm{U}$ and provide precise and detailed data.

The authors would like to thank A. Fleischmann, L. Gastaldo, S. Kempf and C. Enss from the Kirchhoff-Institut für Physik for providing the MMC chips including the sensors and pick-up coils.

\section{References}

[1] M.-M. Bé, V. Chisté, C. Dulieu, X. Mougeot, F.G. Kondev, A.L. Nichols, X. Huang, B. Wang, Table of radionuclides Vol. 7. Monographie BIPM-5, Bureau International des Poids et Mesures, Sevres, France (2013), also available from http: //www.bipm.org/utils/common/pdf/ monographieRI/Monographie_BIPM-5_Tables_ Vol7.pdf

[2] M.A. Kellett, A.L. Nichols, Library of Recommended Actinide Decay Data, 2011, STI/PUB/1618, International Atomic Energy Agency, Vienna (2013)

[3] V.M. Gorozhankin, M.-M. Bé, Appl. Radiat. Isot. 66, 722-728 (2008)

[4] A. Fleischmann, C. Enss, G. Seidel, Cryogenic particle detection, C. Enss (Ed.). Topics Appl. Phys. 99, 151-216 (2005)

[5] M. Rodrigues, M. Loidl, C. Le-Bret, J. Low Temp. Phys. 167, 510-515 (2012)

[6] A. Burck, S. Kempf, S. Schäfer, H. Rotzinger, M. Rodrigues, T. Wolf, L. Gastaldo, A. Fleischmann, C. Enss, J. Low Temp. Phys. 151, 337-344 (2008)

[7] J.L. Campbell, T. Papp, X-Ray Spectrom. 24, 307-319 (1995)

[8] M. Rodrigues, P. Cassette, M.C. Lépy, M. Loidl, Y. Ménesguen, Appl. Radiat. Isot. 109, 500-506 (2016)

[9] U. Schotzig, Nucl. Instrum. Methods Phys. Res. A286, 523 (1990)

[10] D.D. Cohen, Nucl. Instrum. Methods Phys. Res. A267, 492 (1988)

[11] C.J. Bland, J. Morel, E. Etcheverry, M.-C. Lepy Nucl. Instrum. Methods Phys. Res. A312, 323 (1992)

[12] M.-C. Lepy, J. Plagnard, L. Ferreux, Appl. Radiat. Isot. 66, 715 (2008)

[13] V.P. Chechev, N.K. Kuzmenko, Appl. Radiat. Isot. 68, 1578 (2010) 